\title{
Investigation of electron-vibrational relaxation in iodine molecule by means of two-colour laser excitation
}

\author{
M.E. ABRAMENKO, N.K. BIBINOV, A.M. IZMAILOV and D.B. KOCH
}

Physical Research Institute, 198904 St. Peterburg, Russia

One of the problens appearing at the spectroscopic investigation of iodine molecule is to study mechanisms and efficiency of electron-vibrational energy relaxation. This investigation may be interesting both from scientific and applied points of view: it can help in content optimizing of gaseous mixture used as an active medium of lodine lasers, for example. As a result of specific features of the iodine molecule"s potential curves only high vibrational levels of $D Q_{k}^{+}$state ( $V^{\prime}=$ $100+200$ ) can be populated (and consequently investigated) by one photon excitation [1]. The aim of this paper is investigation of mechanisms and efficiency of electron-vibrational energy relaxation in iodine molecule with aid of two-colour laser photoexcitation.

For this problem decision a polychromatic dye laser was developed which is emitting simultaneously two wavelengths independently tunable inside the broad spectral region demanded for this experiment. The active medium is pumped by commercial nitrogen laser. Radiation of both wavelengths is focused inside the cell with pure iodine vapour or its mixture with buffer gas.

Luminescence spectra of $\mathrm{I}_{2}$ molecules is recorded be-

ing excited by the radiation of various sets of wavelengths (i.e. for different levels initial for relaxation) and for various pressure of buffer gas (i.e. for different efficiency of excited levels decay). Registered luminescience spectra were compaired with computer simulated ones. Recorded luminescience spectrum of iodine vapour excited by the light of $\lambda_{1}=$ $560 \mathrm{~nm}$ and $\lambda_{2}=420 \mathrm{~nm}$ simultaneously $(1),(2)$ is shown in fig. 1.

$$
\begin{aligned}
& I_{2}\left(X O_{E}^{+}\right)+h \nu_{1}-\rightarrow I_{2}\left(B O_{2}^{+}\right) \\
& I_{2}\left(B O_{2}^{+}\right)+h \nu_{2}-\rightarrow I_{2}\left(E O_{E}^{+}\right) \text {or } I_{2}\left(\beta I_{g}\right)
\end{aligned}
$$

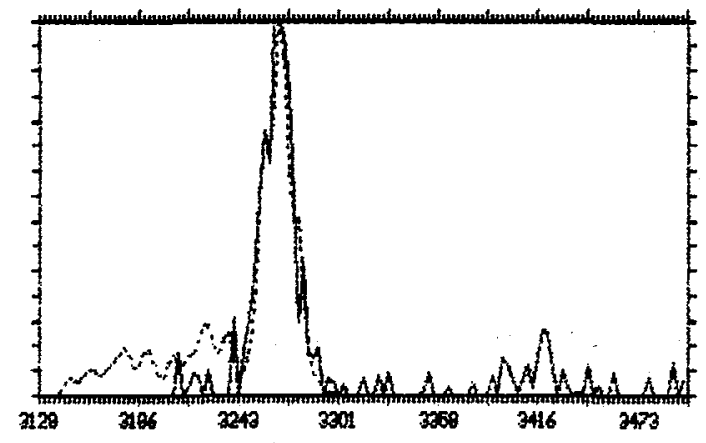

Fig.1. Luminescence spectrum of iodine molecules at two-colour excitation $\left(\lambda_{1}=560 \mathrm{~nm}, \lambda_{2}=420 \mathrm{~nm}\right.$ ). 
A strong transition at $\lambda \sim 326 \mathrm{~nm}$ is observed. According to selections rules for dipole transitions only $I_{2}\left(D O_{a-1 X O E}^{+}\right.$) transition is permitted in this spectral region. Excitation of Dot state from $B O^{+}$is forbidden as known. So it is interesting to find out the reason of mentioned band appearence in the iodine vapour luminescence spectrum at the conditions of our experiment. We discussed three possibilities:

i) Iuminescence from DOt state, being populated by two step photoexcitation through another state, formed as a result of iodine atoms recombination;

ii) luminescence from DOt state, populated by two step photoexcitation through $B O^{+}$state perturbed by the state of other symmetry;

iii) luminescence from Dot state, populated by collisionally induced transitions from EOt state.

Polychromatic laser used in our expirements permits to vary independently the intensity of each lasing line. This gives an ability to measure the dependence of luminescence intensity of iodine vapour $(\lambda \sim 326 \mathrm{~nm})$ on the intensity of excited light $\left(\lambda_{1}=560 \mathrm{~nm}\right.$ and $\left.\lambda_{2}=420 \mathrm{~nm}\right)$. Experimental data show, that the intensity of luminescence IL $(\lambda \sim 326 \mathrm{~nm})$ is a linear function of the excited light intensity (of both lines). Supposition that process (i) is resposible for this luminescence appearence contradicts to this experimental result because both dissociation of iodine molecule and excitation from the intermediate state would be forced by the light $\lambda=420 \mathrm{~nm}$. It should lead, at first, to square dependence of the luminescence intensity from $D$ state on the intensity of the laser beam $\lambda=420 \mathrm{~nm}$; at second - to the independence of IL ( $\lambda_{-}$ $326 \mathrm{~nm}$ ) the intensity of the second laser beam $\lambda=560 \mathrm{~nm}$.

Populating of Dot state, stimulated by collisions with iodine molecules in process (ii) is unlikely probable reason of $\mathrm{I} 2 \mathrm{Iiminescence}(\lambda \sim 326 \mathrm{~nm})$. The pressure of iodine vapour in the experiment was $P\left(T_{z}\right)=0.2$ Torr, so estimated probability of this process is not higher than $3 * 10 \mathrm{~s}^{-1}$. This value is ten times less than the least probability of radiative transition from ion-pair states. More than that, according to the literature data, the lowest ion-pair state $D^{\prime} 2 \mathrm{~g}$ is populated most effectively in the interconversion process even for very smal1 pressure of relaxator. The radiative transition from this state is situated at $\lambda \sim 342 \mathrm{~nm}$. In our experiments the luminescence of lodine vapour in this spectral region is weak (see fig.1).

So the most probable reason of the luminescence at $326 \mathrm{~nm}$ appearence is two-step photoexcitation of pot-state of iodine molecule through BOt-state, mixed with the state of the other symmetry ( $a 1_{\mathrm{g}}$ or $\mathrm{a}^{\prime} \mathrm{O}_{\mathrm{s}}^{+}$). These states can couple through the hyperfine nuclear magnetic interaction.

When two-step photoexcitation is used of the iodine vapour in mixture with helium an intensive band $I_{2}\left(D^{\prime} 2 \alpha^{-} \rightarrow A^{\prime} 2 u\right)$ appears with $\lambda \sim 342 \mathrm{~nm}$ (see fig.2). Increase of $D-->X$ luminescence intensity $(\lambda \sim 326 \mathrm{~nm})$ is observed also, which is forced by processes of electron relaxation from the upper states. But with the increase of noble gas pressure the decrease of $\mathrm{D}--3 \mathrm{X}$ luminescence is observed. It is connected with collisionally induced relaxation from D-state.

Consider main processes, connected with energy relaxation of the excited iodine molecule at the two-step population of $\mathrm{EO}_{\mathrm{\alpha}}^{+}, \mathrm{DO}, \mathrm{W}_{\mathrm{\alpha}}^{+}$states. 


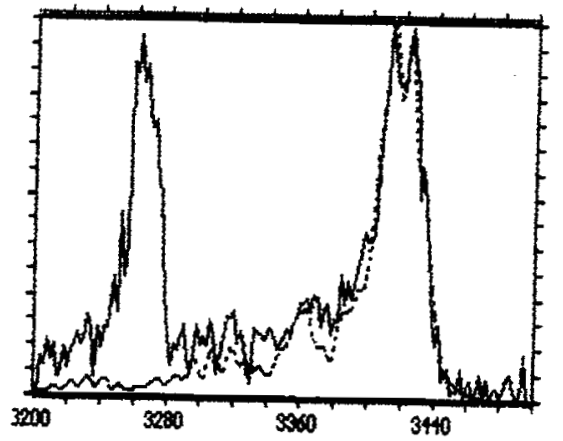

Fig.2. Luminescence spectrum of Is in mixture with helium at two-colour photoexcitation $\left(\lambda_{2}=560 \mathrm{~nm}, \lambda_{2}=420 \mathrm{~nm}, \mathrm{P}_{\mathrm{Ha}}=\right.$ 40 Tor ) : solid line is a measured spectrum, dashed line is a caloulated spectrum.

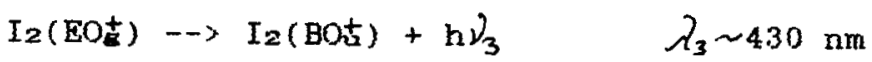

$$
\begin{aligned}
& \mathrm{I}_{2}\left(\mathrm{EO}_{\mathrm{E}}^{+}\right)+\mathrm{He}-\rightarrow \mathrm{I}_{2}(\mathrm{DOH})+\mathrm{He} \\
& I_{2}\left(E O_{k}^{+}\right)+\mathrm{He}-\rightarrow I_{2}\left(\beta 1_{\alpha}\right)+\mathrm{He} \\
& \mathrm{I}_{2}\left(\mathrm{EO}_{\mathrm{E}}^{+}\right)+\mathrm{He}--\mathrm{I} \mathrm{I}_{2}\left(\mathrm{D}^{-2} \mathrm{2}_{\mathrm{s}}\right)+\mathrm{He} \\
& \mathrm{Iz}\left(\mathrm{DO}_{\mathrm{L}}^{+}\right)-\mathrm{I2}\left(\mathrm{XO}_{\mathrm{E}}^{+}\right)+\mathrm{h} \nu_{7} \quad \lambda_{7} \sim 325 \mathrm{~nm} \\
& \left.I_{2}\left(D O_{u}^{+}\right)+\mathrm{He}--\right) I_{2}\left(\beta I_{s}\right)+\mathrm{He} \\
& \mathrm{I}_{2}\left(\mathrm{DO}_{+}^{+}\right)+\mathrm{He} \rightarrow \mathrm{I}_{2}\left(\mathrm{D}^{-} \mathrm{Z}_{\mathrm{E}}\right)+\mathrm{He} \\
& I_{2}\left(\beta I_{a}\right) \rightarrow I_{2}\left(A I_{u}\right)+h \nu_{10} \quad \lambda_{10} \sim 340 \mathrm{~nm} \\
& \mathrm{Iz}\left(\beta \mathrm{I}_{e}\right)+\mathrm{He} \rightarrow \mathrm{Ia}\left(\mathrm{D}^{+} \mathrm{Z}_{e}\right)+\mathrm{He} \\
& I_{2}\left(D^{*} 2_{B}\right) \rightarrow I_{2}\left(A^{*} 2_{u}\right)+h \nu_{12} \quad \lambda_{12} \sim 342 \mathrm{~nm}
\end{aligned}
$$

According to literature data it is possible to neglect the processes of nonradiational decay of ion-pair states in collisions with helium. Luminescence intensities for corresponding transitions can be written in the following form:

$$
\begin{aligned}
& \mathrm{ID}_{\mathrm{D}}-x \mathrm{x}=\left(\mathrm{ND}+[\mathrm{E}] \widetilde{2 m K}_{4}[\mathrm{He}]\right) \mathrm{Kr} /\left(\mathrm{Kr}+\left(\mathrm{KB}_{\mathrm{B}}+\mathrm{K}_{\theta}\right)[\mathrm{He}]\right) \\
& I_{D-D A}=\left[\mathrm{He}_{0}\right]\left[[\beta] \tau_{\beta} \mathrm{K}_{11}+[\mathrm{D}] \tau_{\mathrm{DK} \theta}+[\mathrm{E}] \tau_{\mathrm{EK} B}\right)
\end{aligned}
$$

Comparing the intensities $I_{2}(D-\rightarrow X)$ and $I_{2}\left(D^{*} \rightarrow A^{*}\right)$ extrapolated to zero helium pressure, (see fig.3) we find out, that not more than $20 \%$ of the energy of excitation pass through $D-$ state. The rate constant of collisionaly induced excitation energy decay from E-state can be measured if the radiative lifetime of this state is known. It is known from $[2,3]$ that $\mathrm{K}_{3}=(3.6 \pm 0.3) * 10^{7} \mathrm{~s}^{-1}$. The value of $K_{4}+K_{6}+K_{8}$ measured in our work is $(1.9 \pm 0.5) * 10^{-11} \mathrm{~s}^{-1} \mathrm{~cm}^{3} \mathrm{~mol}-1$. The rate constant of interconversion from D-state ( $\mathrm{Ka}+\mathrm{Ka}$ ) messured with account of radiative lifetime of $D$ state $K_{T}=7.7 * 10^{7} s^{-1}$ equals $(1.4 \pm 0.1)$ *10-12 $\mathrm{s}^{-1} \mathrm{~cm}^{2} \mathrm{~mol}{ }^{-1}$. The value of interconversion constant 


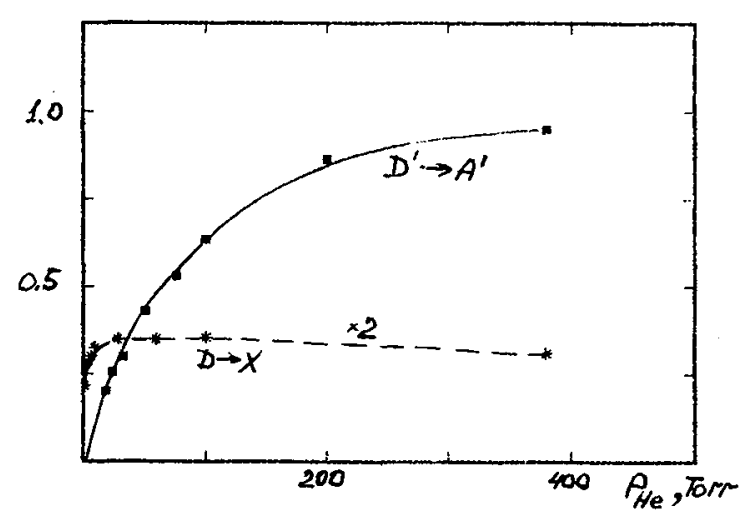

Fig.3. Intensity of $\mathrm{I}_{2}\left(D^{-} \rightarrow->A^{\prime}\right)$ and $\mathrm{I}_{2}\left(\mathrm{D}-\rightarrow A_{)}\right)$luminescence $\mathrm{a}_{3}$ a function of He pressure $\left(\lambda_{1}=560 \mathrm{~nm}, \lambda_{2}=420 \mathrm{~nm}\right)$.

$\mathrm{K} 8+\mathrm{K} \theta$ measured at two-colour photoexcitation $\lambda_{1}=585 \mathrm{~nm}, \lambda_{2}=$ $420 \mathrm{~nm}$ is equal to $(6 \pm 1) * 10^{-12} \mathrm{~s}^{-1} \mathrm{~cm}^{3} \mathrm{~mol}^{-1}$. This is approximately 4 times higher than in the case of lodine molecule excitation through $E$ state.

Different electron-vibrational levels of ion-pair states were selectively populated with aid of polychromatic dye laser. Population of Dou-state is observed at two-colour photoexcitation through Bot-state, which is connected with mixing of the last state of other symmetry. Rate constants of electronvibrational relaxation from $D$ - and $E$-states are measured in mixture of iodine vapour with helium.

Authors express acnoledgement to Russian foundation of Basic Research for the support of this work.

\section{Literature}

1. Mulliken R.S., J.Chem.Phys., 1971, y.55, p.288-309.

2. J.P.Perrot, B.Femelat, J.L.Subti1, M.Broyer, J.Chevaleyre, Mol. Phys., 1987, y.61, n.1, p.85-95.

3. P.I.Jensbury, K.P.Lawley, T.Ridley et al, Chem.Phys., 1991, 151 , p.103-109. 